\title{
Calidad de vida de adultos con discapacidad intelectual institucionalizados en Chile desde la perspectiva de los proveedores de servicios*
}

\section{Quality of Life of Adults with Intellectual Disabilities Institutionalized in Chile from the Perspective of Service Providers}

Recibido: julio 6 de 2011| Revisado: octubre 28 de 2012 | Aceptado: diciembre 20 de 2012

\author{
VANESSA Vega CÓRDOVA ** \\ Pontificia Universidad Católica de Valparaíso, Chile \\ CRISTINA JENARO RÍO **** \\ NOElia FlORES ROBAINA**** \\ Universidad de Salamanca, España \\ MARIBEL CRUZ ORTIZ ****** \\ Universidad Autónoma de San Luis Potosí, México. \\ CARMEN ARTAZA ******* \\ Corporación de Ayuda al Niño Limitado, Chile
}

doi:10.11144/Javeriana.UPSY12-3.cvad

Para citar este artículo: Vega, V., Jenaro, C., Flores, N., Cruz, M. \& Ataza, C. (2013). Calidad de vida de adultos con discapacidad intelectual institucionalizados en Chile desde la perspectiva de los proveedores de servicios. Universitas Psychologica, 12(3), 923-932. doi:10.11144/Javeriana.UPSY12-3.cvad

* Agradecimientos: A la Fundación Coanil, por su colaboración y participación en la investigación.

** Pontificia Universidad Católica de Valparaíso, Chile. Doctora en Educación, Universidad de Salamanca, España. Profesora de Educación Diferencial, Escuela de Pedagogía, Researcher ID: H-6680-2013. Autora para la correspondencia: Avda. El Bosque 1290, Santa Inés. Viña del Mar (Chile). Tel: +56 32-2667329. E-mail: vanejuay@ gmail.com

**** Universidad de Salamanca, España. Doctora en Psicología, INICO. Facultad de Psicología, Researcher ID: H-6386-2013. Avda. De la Merced, 109-131, 37005 Salamanca (España). Tel: +34 -923- 294 695. Fax: +34 -923- 294 685. E-mail: crisje@usal.es

***** Universidad de Salamanca, España. Doctora en Psicología, INICO. Facultad de Psicología, Researcher ID: H-7492-2013. Avda. De la Merced, 109-131, 37005-Salamanca (España). Tel: +34 -923- 294 695. Fax: +34-923-294 685. E-mail: nrobaina@usal.es

******** Universidad Autónoma de San Luis Potosí, México. Doctora en Enfermería por la Universidad de Salamanca, España. Researcher ID: H-79712013. E-mail: redazul@hotmail.com

******** Directora Área Técnica, Fundación Coanil. Julio Prado 1761, Ñuñoa, Santiago de Chile. E-mail: cartaza@coanil.cl

\section{RESUMEN}

El presente estudio de corte cualitativo, pretende analizar la percepción de profesionales, cuidadores de trato directo y directores sobre la calidad de vida de los usuarios de centros residenciales de Chile. La recogida de información se ha realizado mediante grupos focales. Los resultados obtenidos indican diferencias en las percepciones sobre la calidad de vida de las personas con discapacidad intelectual o del desarrollo según la tipología de informante. Por otro lado, los comentarios sobre la calidad de vida de los residentes reflejan una actitud excesivamente protectora que reduce las posibilidades de que estas personas sean los actores principales de sus vidas. Esta aproximación cualitativa ha permitido también identificar dimensiones del modelo de calidad de vida que se ven obstaculizadas por la institucionalización, como son los aspectos emocionales y materiales. Este estudio acentúa la importancia de los modelos mentales sustentados por los profesionales y su reflejo en las prácticas promotoras de la calidad de vida. Palabras clave autores

Discapacidad intelectual, investigación cualitativa, calidad de vida, institucionalización.

Palabras clave descriptores

Grupos focales, psicología de la salud, Chile.
A B S T R A C T
This qualitative study aims to analyze the perception of professionals, direct- care assistants, and managers on quality of life of residential users in Chile. Information was gathered by means of focus groups. The results indicate differences in perceptions of quality of life of people with intellectual or developmental disabilities by type of informant. Comments on the quality of life of the residents reflect overly protective attitudes that reduce the chances of being the main actors of their lives. This qualitative approach also allowed the identification of dimensions of the quality of life model which are hampered by institutionalization, such as emotional and material well- being. This study emphasizes the importance of mental models supported by professionals and its reflection on the practices that promote quality of life. Key words authors Intellectual disability, quality of life, institutionalization, qualitative research. Key words plus Focus Groups, Health Psychology, Chile. 
En la actualidad el concepto de calidad de vida es utilizado como una noción sensibilizadora que proporciona una referencia y orientación desde la perspectiva del individuo centrado en la persona y en su ambiente. También se emplea como constructo social para mejorar el bienestar de la persona y contribuir al cambio social. Además el concepto de calidad de vida constituye un tema unificador que otorga un lenguaje común y un marco sistemático para aplicar conceptos y principios asociados (Luckasson et al., 2002). Cuando hablamos de calidad de vida nos referimos (Schalock \& Verdugo, 2007) a un estado deseado de bienestar personal que es multidimensional, tiene propiedades éticas o universales y émicas o ligadas a la cultura, con componentes objetivos y subjetivos y está influenciada por factores personales y ambientales.

Aunque existen diversas concepciones de este concepto, el modelo de calidad de vida por el que apostamos es el propuesto por Schalock y Verdugo (2003), ya que aborda desde una perspectiva de sistemas su planteamiento conceptual. Otro aspecto esencial del modelo es su pluralismo metodológico. Esto supone defender la necesidad de combinar procedimientos cuantitativos y cualitativos en la evaluación e investigación para medir la perspectiva personal y permitir la evaluación funcional y de los indicadores sociales. Esto es aún más importante si tenemos en cuenta las limitaciones de una evaluación meramente cuantitativa. Así, recientes revisiones sistemáticas sobre instrumentos de evaluación de la calidad de vida llaman la atención sobre la necesidad de continuar trabajando para desarrollar instrumentos adecuadamente validados, e incluso cuestionan la utilidad de los existentes con población con discapacidad intelectual más severa o problemas de comportamiento relacionados (Townsend-White, Pham \& Vassos, 2012).

El modelo de calidad de vida supone una guía importante para la evaluación de resultados personales, la planificación de servicios para agencias y proveedores y la puesta en práctica de estrategias de mejora de la calidad de dichos servicios. Es también un marco de referencia para evaluar el impacto de políticas sociales (Gómez, Arias, Verdugo \& Navas, 2012; Schalock, Keith, Verdugo \& Gómez,
2010; Schalock, Verdugo, Bonham, Fontava \& Van Loon, 2008). Por ejemplo, recientes revisiones sobre las políticas de desinstitucionalización de personas con discapacidad intelectual que han tenido lugar a nivel mundial indican que la reubicación en la comunidad ha tenido un impacto general positivo en la calidad de vida de muchos participantes. Sin embargo, muchos estudios han revelado que las principales mejoras tuvieron lugar a corto plazo, encontrándose un estancamiento a medio y largo plazo. Además, los participantes continuaron mostrando bajos niveles de integración en la comunidad. También parece que las necesidades relacionadas con la salud no están siendo adecuadamente atendidas en la comunidad. Una de las limitaciones de muchos de estos estudios es que, por lo general, emplean tan solo medidas objetivas de evaluación de la calidad de vida, con una clara ausencia de evaluaciones subjetivas o desde el punto de vista de los implicados (Chowdhury \& Benson, 2011).

El interés por conocer la calidad de vida de las personas con discapacidad ha permitido el desarrollo de las diferentes estrategias de recogida de información para obtener una medida fiable de este constructo. Para Dennis, Williams, Gisngreco y Cloninger (1993), los enfoques que han guiado la evaluación de la calidad de vida son variados, pero se pueden agrupar en cuantitativos y cualitativos. Dentro del ámbito cuantitativo, en residencias para personas con discapacidad intelectual, las investigaciones en calidad de vida llevan siendo objeto de estudio desde hace una década, aproximadamente. Entre ellas destacan las investigaciones de Felce y Emmerson (2001) o de Stancliffe, Hayden, Larson y Lakin (2002), o los estudios centrados en analizar la relación entre servicios residenciales y la calidad de vida de sus residentes (Hatton, Emerson, Robertson, Herdenson \& Cooper, 1995; Keith \& Rawlinson, 1998; Rouke, Grey, Fuller \& MacClean, 2004; Stancliffe, 2002; Young, 2006), una medición basada en los resultados de los servicios.

Como plantea Giné (2004), la calidad de vida constituye un reto para diseñar y poner en práctica políticas sociales que involucren a personas con discapacidad intelectual, tanto para el diseño de las condiciones de vida y programas de apoyo 
para personas y grupos como para la evaluación de los servicios. En este sentido, en Chile se han llevado a cabo algunas investigaciones de corte cuantitativo para evaluar y medir la calidad de vida objetiva de residentes de instituciones residenciales permanentes. Los resultados ponen de manifiesto la existencia de puntuaciones más bajas en las dimensiones de calidad de vida relacionadas con la inclusión social y los derechos. Por otro lado, una mayor severidad de la discapacidad se ha encontrado asociada con puntuaciones significativamente inferiores en calidad de vida. La variable institucionalización no parece afectar diferencialmente los resultados en calidad de vida (Vega, Jenaro, Cruz \& Flores, 2011).

En el ámbito de investigación cualitativa, recientes investigaciones sobre calidad de vida la vida en residencias (Holburn, Cea, Coull \& Goode, 2007) encuentran diferencias en la percepción de la calidad de vida de personas con discapacidad intelectual, dependiendo de si los encuestados son dichas personas, los familiares, los cuidadores o los directores de centros. Dado que visiones diversas pueden suponer diferentes posturas sobre prioridades a la hora de proveer de servicios, es importante analizar si tales diferencias se encuentran también en nuestro contexto cultural o en contextos hispanohablantes. Ello permitirá poner en marcha estrategias para armonizar políticas y prácticas orientadas a mejorar la calidad de vida, en la línea de lo planteado por Schalock y Verdugo (2003).

Así pues, la presente investigación de corte cualitativo tiene como finalidad:

1. Conocer la percepción de profesionales, cuidadores de trato directo y directores de los servicios residenciales sobre la calidad de vida de los usuarios de centros residenciales.

2. Identificar semejanzas y diferencias en estas percepciones en función de la tipología del informante.

En el presente estudio esperamos encontrar: 1) diferencias en la percepción de la calidad de vida en función de los informantes y 2) impacto de la institucionalización en los juicios sobre calidad de vida.

\section{Método}

\section{Participantes}

Los participantes de esta investigación fueron trabajadores chilenos pertenecientes a seis hogares de la Fundación Coanil, Corporación de Ayuda al Niño Limitado, de las regiones Quinta y Metropolitana, seleccionados según tres perfiles de interés: directores de centros, profesionales de diferentes áreas y cuidadores de trato directo. Los criterios de inclusión fueron: 1) llevar trabajando más de un año en la institución, 2) conocer a las personas con discapacidad objeto de evaluación por más de tres meses y 3) otorgar su consentimiento informado, tras garantizarles la confidencialidad de la información recogida.

Los tres grupos se constituyeron de la siguiente manera: (1) el primero, compuesto por miembros de los equipos multiprofesionales: tres psicólogos, tres trabajadoras sociales y un kinesiólogo; (2) el segundo, conformado por nueve cuidadores de trato directo y (3) un tercer grupo compuesto por los seis directores de los diferentes centros, tres psicólogos, dos educadores diferenciales y un terapeuta ocupacional.

\section{Procedimiento}

Los tres grupos focales se llevaron a cabo durante los meses de octubre y noviembre de 2009. Se empleó la modalidad de sesiones de grupo tradicionales, para lo cual se elaboró un guión que contenía las preguntas estímulos y las indicaciones para iniciar y cerrar la sesión. El guión se centraba en la calidad de vida individual y la percibida por parte de los prestadores de servicios sobre las personas con discapacidad que viven en las residencias de la fundación. Concretamente, se pretendió responder a dos interrogantes: ¿Qué es para ustedes experimentar calidad de vida? y iEn qué medida cree que los residentes de los centros experimentan calidad de vida y cómo se refleja esta?

Las transcripciones se realizaron en formato Word, teniendo presente la omisión de cualquier dato que pudiese identificar a cada uno de los par- 
ticipantes, para respetar así el compromiso con el anonimato de la información recogida. La codificación fue realizada por cuatro investigadores independientes, en el mes de abril de 2010. El análisis de la concordancia de sus valoraciones se llevó a cabo mediante el estadístico alfa de Krippendorff, además de otros estadísticos de acuerdo entre jueces (kappa de Fleiss, kappa de Cohen y porcentaje medio de acuerdo entre pares de jueces). El estadístico alfa de Krippendorff cuenta entre sus ventajas el hecho de que puede emplearse con cualquier escala de medida, admite cualquier número de evaluadores, puede calcularse con independencia de que existan datos perdidos y satisface los criterios más importantes que debe tener en cuenta cualquier medida de fiabilidad (Verdugo, Arias \& Ibáñez, 2007).

Los análisis de las unidades de análisis y los códigos fueron realizados por cuatro jueces expertos de diferentes áreas, dos del área de educación, uno del área de salud y uno del área de psicología, a quienes se les hizo llegar un documento con las transcripciones de los diferentes grupos focales y las unidades de análisis seleccionadas. El proceso se desarrolló mediante un análisis de contenido, siguiendo las etapas del modelo por pasos del desarrollo deductivo-inductivo propuesto por Mayring (2000).

El referente teórico para la codificación de la información es el modelo de calidad de vida de Schalock y Verdugo (2003), compuesto por ocho dimensiones: bienestar emocional, relaciones interpersonales, bienestar material, desarrollo personal, bienestar físico, autodeterminación, inclusión social y derechos. A estas dimensiones hemos se añadió la valoración de la institucionalización, para determinar en qué medida las circunstancias en que se encuentran estas personas con discapacidad intelectual pueden afectar los juicios sobre su calidad de vida.

La integración y descripción de los hallazgos requirió varios pasos. En primer lugar, se seleccionaron los fragmentos textuales más relevantes de cada participante y grupo. En segundo lugar, se realizó un análisis descriptivo de los perfiles de los grupos y se obtuvo la distribución de frecuencias para cada indicador del paradigma de apoyos. En tercer lugar, se analizó la posible existencia de asociaciones significativas entre variables categóricas (i. e., grupo profesional, centro de adscripción) y frecuencia de mención de unos u otros indicadores de apoyos. Todo ello se expone con más detalle a continuación.

\section{Resultados}

Los resultados se exponen en dos partes, una primera parte dice relación con los resultados de la fiabilidad de las codificaciones de calidad de vida; los análisis muestran niveles de acuerdo satisfactorios entre evaluadores, con un valor promedio de $69.35 \%$ y valores que oscilan entre $61.7 \%$ y $90.6 \%$, que pueden considerarse satisfactorios. El estadístico kappa de Cohen osciló entre 0.55 y 0.89, con un valor medio de 0.64. El estadístico kappa de Fleiss fue de 0.64 (acuerdo observado $=0.69$; acuerdo esperado $=0.15)$, igualmente adecuado. El estadís tico alfa de Krippendorff, por su parte, fue $=0.64$.

Uno de los objetivos del estudio se centraba en analizar los conceptos personales de calidad de vida ("¿Qué es para ustedes experimentar calidad de vida?”) y sus posibles diferencias frente a las estimaciones de calidad de vida de terceros (" $i C r e e$ que los residentes experimentan calidad de vida, y por qué?"). Así pues, en la Tabla 1 se plasman los principales resultados donde puede observar que existen diferencias en las apreciaciones. Concretamente, en primer lugar, los proveedores de servicios consideran la calidad de vida como algo global y que se ve influida por aspectos primordiales de siete de las ocho dimensiones del modelo (no se considera la inclusión social). En segundo lugar, los encuestados destacan la importancia de sentirse bien emocional y físicamente, y remarcan la importancia de la autodeterminación en sus vidas. Sin embargo, a la hora de valorar la vida de los residentes, aunque se mencionan todas las dimensiones, el contenido de los comentarios reflejan una actitud excesivamente protectora y donde las personas con discapacidad intelectual y del desarrollo no son los actores principales de sus vidas.

El análisis de las transcripciones permite también identificar un tema de discusión importante que aparece reiteradamente en los diálogos de los provee- 
TABla 1

Concepto y percepción de calidad de vida por parte de los proveedores de servicios

\begin{tabular}{|c|c|c|}
\hline Dimensiones de Calidad de vida & Concepto de calidad de vida & $\begin{array}{c}\text { Valoración de la calidad de vida de los } \\
\text { residentes }\end{array}$ \\
\hline Bienestar Emocional & $\begin{array}{l}\text { "Siento que la calidad de vida es todo } \\
\text { aquello que nos genera a nosotros } \\
\text { satisfacción, felicidad y bienestar". } \\
\text { "Lo más importante de la calidad de } \\
\text { vida, es cómo yo me estoy sintiendo } \\
\text { con la cosas que me pasan". } \\
\text { "Calidad de vida es estar bien, sentir- } \\
\text { me cómoda, a gusto conmigo misma, } \\
\text { estar cómoda con lo que hago". }\end{array}$ & $\begin{array}{l}\text { "Es enseñarles cómo quererse, cómo cui- } \\
\text { darse a ellas mismas". } \\
\text { "Yo veo que ha mejorado mucho lo con- } \\
\text { ductual, ellas se ven felices". } \\
\text { "Para darles una buena calidad de vida es } \\
\text { que se quieran". }\end{array}$ \\
\hline Relaciones Interpersonales & $\begin{array}{l}\text { "Compartir con los amigos". } \\
\text { "Estar bien con el núcleo familiar y en } \\
\text { el trabajo". } \\
\text { "Tener buenas relaciones con las per- } \\
\text { sonas, saber aceptar a los demás". }\end{array}$ & $\begin{array}{l}\text { "Cuando tienen la posibilidad de salir a } \\
\text { grupos de la tercera edad". } \\
\text { "La familia es elemental y siempre van a } \\
\text { tener la tristeza de que no los vayan a ver } \\
\text { (...) podemos compensar pero a la fami- } \\
\text { lia no la podemos reemplazar". }\end{array}$ \\
\hline Bienestar Material & $\begin{array}{l}\text { "Creo que es algo superintegral, que } \\
\text { tiene que ver con los medios, con las } \\
\text { condiciones de vida". }\end{array}$ & $\begin{array}{l}\text { "El hecho de que tengan sus cosas pro- } \\
\text { pias los hace felices". } \\
\text { "Ellos experimentan calidad de vida } \\
\text { teniéndoles una buena cama, los medica- } \\
\text { mentos a las horas". } \\
\text { "Teniendo su alimentación, su vestimen- } \\
\text { ta". }\end{array}$ \\
\hline Desarrollo Personal & $\begin{array}{l}\text { "La necesidad de realización perso- } \\
\text { nal, profesional, de recreación de } \\
\text { salud y espiritual". } \\
\text { "Poder desenvolverme y usas mis } \\
\text { habilidades". }\end{array}$ & $\begin{array}{l}\text { "Ellas sintiéndose útiles son felices". } \\
\text { "La calidad de vida la han experimentado } \\
\text { quienes van a trabajar". } \\
\text { "Cuando logramos que un chico salga y } \\
\text { vuelva con horario". }\end{array}$ \\
\hline Bienestar Físico & $\begin{array}{l}\text { "Para la calidad de vida es importan- } \\
\text { tísimo tener salud". } \\
\text { "Tener una buena salud mental". }\end{array}$ & $\begin{array}{l}\text { "Que tengan sus medicamentos, los mé- } \\
\text { dicos..., porque como ellos no se comu- } \\
\text { nican". } \\
\text { "Enseñándoles por ejemplo cómo deben } \\
\text { comer, la obesidad". }\end{array}$ \\
\hline Autodeterminación & $\begin{array}{l}\text { "En la calidad de vida también es } \\
\text { importante que uno tenga la opción } \\
\text { de elegir". } \\
\text { "Que esta vida que tengo, responda } \\
\text { a mis intereses, a mis necesidades y a } \\
\text { mi motivación". }\end{array}$ & $\begin{array}{l}\text { "Yo los veo felices cuando pueden decidir } \\
\text { qué es lo que comen, distribuir su dine- } \\
\text { ro". } \\
\text { "El tema de sentirse que andan solos, de } \\
\text { sentirse independientes, que tu les das la } \\
\text { confianza". } \\
\text { "Siento que la población adulta que está } \\
\text { en los hogares no tuvo y no tiene la posi- } \\
\text { bilidad de optar". }\end{array}$ \\
\hline Inclusión Social & & $\begin{array}{l}\text { "Buscamos alternativas en la comuni- } \\
\text { dad". } \\
\text { "Tratamos de que salgan en grupo, aun- } \\
\text { que sea por el alrededor". } \\
\text { "Tratamos de que las personas adultas } \\
\text { puedan salir". }\end{array}$ \\
\hline Derechos & $\begin{array}{l}\text { "El poder optar de la equidad, de la } \\
\text { justicia son cosas que van de la mano } \\
\text { de calidad de vida". } \\
\text { "Tiene que ver con los derechos bási- } \\
\text { cos de ser persona". }\end{array}$ & $\begin{array}{l}\text { "El tema de sus derechos es bien difícil". } \\
\text { "Hay una vulneración de derechos, hay } \\
\text { algo que está por medio". } \\
\text { "Nos estamos posicionando más en nues- } \\
\text { tro deber de exigir sus derechos". }\end{array}$ \\
\hline
\end{tabular}

Fuente: elaboración propia. 
dores de servicios, como es el hecho de que la calidad de vida se ve afectada por la institucionalización. En este sentido y centrándose en el análisis de posibles diferencias en función del perfil profesional, para los profesionales, el bienestar físico y el hecho de que las personas se encuentren sanas son dos de los fines de estas instituciones. Sin embargo, se plantean contradicciones en la dimensión de bienestar material ya que se incide en la necesidad de realizar cambios en cuanto a los sistemas de pabellones y a la falta de espacio, pero se reconoce al mismo tiempo que no se cuenta con los medios para llevar a efecto dichos cambios. Esto queda reflejado en opiniones que aluden a: "hay un tema de identidad, de independencia (...); el sistema de pabellones tampoco cumple con las necesidades de tu espacio de intimidad". Esta afirmación se ve apoyada por manifestaciones del tipo: "pero el tema de los espacios, de los pabellones, del televisor, de la ropa que me pongo (...), es todo supercomplejo, todo masivo (...)", o también "el tema de la masificación (...); es necesario contar con más personal especializado".

Para los directores, la dimensión bienestar emocional se asocia o se ve afectada por la institucionalización, aunque se haga lo posible por suplir las carencias afectivas. Esto lo expresan en opiniones tales como: "El tema de la institucionalización, por estar en este sistema, hay cierta población que tiene otro tipo de características, que requieren de una atención más personalizada" o "Cuando hablamos de institucionalización claro que va a haber carencias en cuanto a la calidad de vida, que tal vez no las vamos a poder reemplazar".

En tercer lugar, y por su parte, los cuidadores de trato directo no hacen alusión en sus citas a contenidos referentes al código de institucionalización, lo que refleja la necesidad de trabajar este tema con ellos, y confirma la necesidad de abordar los modelos mentales de los diferentes agentes que trabajan con este colectivo.

En la Figura 1 se presentan los resultados obtenidos respecto a la frecuencia de mención de las dimensiones de calidad de vida y de la institucionalización, agrupados en función de la tipología de proveedores de servicios. Se puede observar cómo, en general, las dimensiones más mencionadas se asocian con el bienestar emocional y la autodeterminación, mientras que las dimensiones menos mencionadas se relacionan con la inclusión social y derechos. En cuanto al análisis de la posible asociación entre tipología del informante y las opiniones expresadas sobre calidad de vida, este reflejó asociaciones significativas entre las variables estudiadas $\left(\chi^{2}=44.262 ; g l=16 ; p<0.001\right)$ (Figura 1). La figura permite apreciar cómo en algunas dimensiones (p. ej., bienestar emocional, autode-

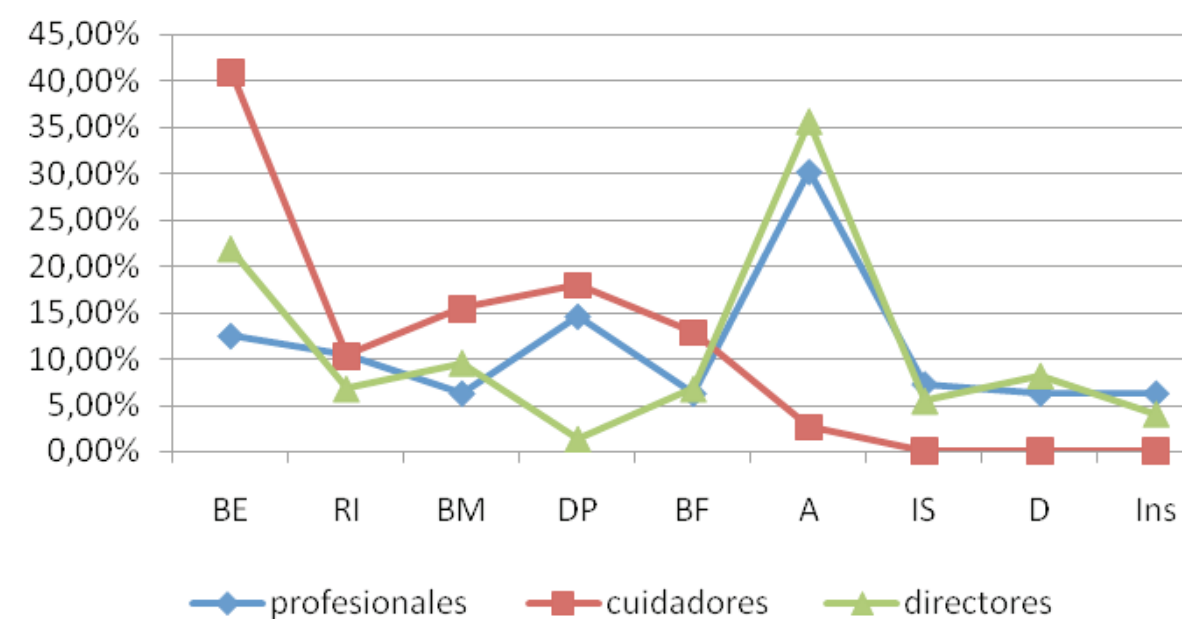

Figura 1. Frecuencia de mención de las dimensiones de calidad de vida según el perfil profesional.

Nota. $\mathrm{BE}=$ Bienestar Emocional; RI = Relaciones Interpersonales; $\mathrm{BM}=$ Bienestar Material; DP = Desarrollo Personal; $\mathrm{BF}=$ Bienestar Físico; A = Autodeterminación; IS = Inclusión Social; D = Derechos; INS = Institucionalización.

Fuente: elaboración propia. 
terminación) existen diferencias más acusadas en cuanto a frecuencia de mención. Sin embargo, en otras dimensiones (p. ej., relaciones interpersonales, bienestar físico), las diferencias son menores.

De modo complementario, en la Figura 2 se ofrecen, en una escala ordinal (indicando el 1 la mayor frecuencia de mención y el 9 la menor), las diferentes dimensiones de calidad de vida consideradas por los entrevistados. Se puede observar por ejemplo cómo las cuidadoras no aluden a dimensiones como derechos o inclusión social, al tiempo que otorgan una gran importancia al bienestar emocional. Por su parte, los directores se refieren a todas las dimensiones pero, a diferencia de los otros dos grupos de informantes, conceden una menor importancia al desarrollo personal y una mayor importancia a la autodeterminación. Los profesionales ofrecen comentarios de igual frecuencia ante dimensiones como la institucionalización, los derechos, el bienestar físico o el bienestar material. Se observa así cómo mientras las cuidadoras centran la valoración de la calidad de vida en el bienestar emocional, los otros dos grupos fijan su mirada en la autodeterminación.

\section{Discusión}

La valoración de la calidad de vida de usuarios de centros residenciales para personas con discapacidad intelectual y del desarrollo en Chile, por medio de la técnica de grupos de discusión o grupos focales, permite recoger en profundidad los diferentes matices de los encuestados. Varios resultados merecen ser destacados. En primer lugar, el hecho de que los juicios sobre lo que constituye una vida de calidad varíen dependiendo del "receptor" de dicha vida. Así, se han identificado diferencias cuando se valora la propia situación frente a cuando se refiere a la calidad de vida de terceros, concretamente de personas con discapacidad intelectual. Ello justifica la necesidad de trabajar por potenciar la calidad de vida de estas personas para que se convierta en realidad el principio planteado por Schalock y Verdugo (2003) de que la calidad de vida está compuesta por las mismas dimensiones e indicadores que son importantes para todas las personas. El hecho de presentar una discapacidad o de encontrarse institucionalizado no se debe traducir en una merma de las expectativas de lo que es una vida de calidad para estas personas.

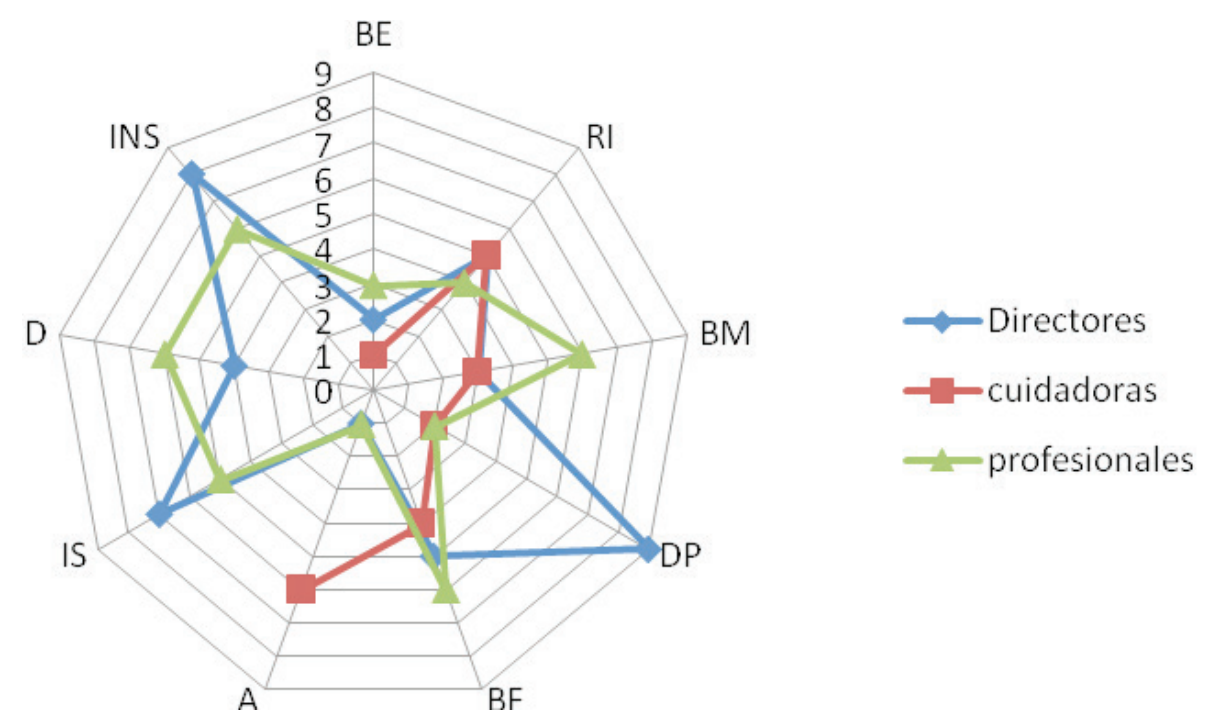

Figura 2. Diferencias en el orden (frecuencia) de mención de las dimensiones de calidad de vida por perfil profesional. Nota. $\mathrm{BE}=$ Bienestar Emocional; RI = Relaciones Interpersonales; BM = Bienestar Material; DP = Desarrollo Personal; $\mathrm{BF}=$ Bienestar Físico; AD = Autodeterminación; IS = Inclusión Social; D = Derechos; INS = Institucionalización. Fuente: elaboración propia. 
En el presente estudio se han encontrado además diferentes percepciones de calidad de vida, dependiendo del tipo de informante, lo que coincide con estudios como los llevados a cabo por Holburn et al. (2007), con empleo de las mismas técnicas. Difiere sin embargo de estudios de corte cuantitativo (Bagnato \& Jenaro, 2010; Córdoba, Gómez \& Verdugo, 2008; Vega, Jenaro, Morillo, Cruz \& Flores, 2011), basados en el mismo modelo de calidad de vida que el empleado en el presente estudio. Esto pone de manifiesto la importancia de emplear metodologías mixtas para poder apreciar una realidad tan compleja como la que nos ocupa. De nuevo, se quiere insistir en la importancia de los modelos mentales sustentados por los profesionales y su reflejo en las prácticas promotoras de la calidad de vida.

Esta aproximación cualitativa ha permitido también identificar dimensiones del modelo de calidad de vida que se ven obstaculizados por la institucionalización, como son los aspectos emocionales y materiales. El impacto de la misma en la calidad de vida, mencionado por profesionales y directores, refuerza la necesidad de generar instancias que permitan a los usuarios de los centros vivir una vida plena. La ausencia de comentarios sobre las implicaciones de la institucionalización por parte de las cuidadoras, refleja también una posible falta de conciencia sobre las potencialidades de este colectivo. Ello justifica la puesta en marcha de actuaciones para fomentar el logro de dicha vida de calidad, por parte de todos los proveedores de servicios.

Como numerosas investigaciones ponen de manifiesto, la desinstitucionalización genera cambios en la vida y en la conducta adaptativa de las personas con discapacidad intelectual (Dunt \& Cummins, 1990; Emerson \& Hatton, 1996). En otras palabras, esta desinstitucionalización mejora su calidad de vida (Kim, Larson \& Lakin, 2001; Mirfin-Veitch, Bray \& Ross, 2003; O`Brien, Tesing, Tuck \& Copie, 2001; Young, Ashsman, Sigafoos \& Grewell, 2001). También es cierto que las políticas de desinstitucionalización han producido cambios drásticos en el tamaño y tipo de residencias donde viven las personas y que cada vez más surgen evi- dencias sobre el impacto de factores ambientales en la promoción del funcionamiento y su calidad de vida (Gómez, Canal \& Verdugo, 2002; Schalock \& Kierman, 1990).

Es por tanto un reto aún pendiente, para muchas organizaciones e incluso países, fomentar una verdadera conciencia sobre la importancia de la igualdad de oportunidades y el trabajo en pro de una vida de calidad en entornos lo más normalizados posible. En los últimos tiempos, a nivel internacional, son innegables los cambios en políticas y prácticas en el ámbito organizacional y sistémico, basados en el marco de la calidad de vida y caracterizados por la prestación de ayudas y servicios, los sistemas de apoyos comunitarios, la gestión participativa, los sistemas de evaluación interna, el uso de metodologías de evaluación y el aumento de la calidad (Schalock et al., 2008). Todo esto hace sentir optimismo sobre la evolución de los servicios en Chile para este colectivo y estimula el trabajo por su mejora continua.

\section{Referencias}

Bagnato, M. J. \& Jenaro, C. (2010). Aplicación de la Escala de Calidad de Vida (Schalock y Keith, 1993) con tres grupos de informantes: evidencias adicionales sobre su utilidad. Siglo Cero, 41(2), 81-98.

Chowdhury, M. \& Benson, B. A. (2011). Deinstitutionalization and quality of life of individuals with intellectual disability: A review of the international literature. Journal of Policy and Practice in Intellectual Disabilities, 8(4), 256-265.

Córdoba, L., Gómez, L. \& Verdugo, M. A. (2008). Calidad de vida familiar en personas con discapacidad: un análisis comparativo. Universitas Pshychologica, 7(2), 369-383.

Dennis, R., Williams, W., Giangreco, M. \& Cloninger, C. (1993). Quality of life as context for planning and evaluation of services for people with disabilities. Exceptional Children, 59(6), 499-512.

Dunt, D. \& Cummins, R. (1990). The deinstitutionalization of St. Nicholas Hospital I: Adaptive behavior and physical health. Australia and New Zealand Journal of Developmental Disabilities, 16(1), 5-18. 
Emerson, E. \& Hatton, C. (1996). Impact of deinstitutionalization on service users in Britain. En J. Mansell \& K. Ericsson (Eds.), Deinstitutionalization and community living (pp. 169-184). London: Chapman \& Hall.

Felce, D. \& Emerson, E. (2001). Living with support in a home in the community: Predictors of behavioral development and household and community activity. Mental Retardation and Developmental Disabilities Research Reviews, 7(2), 75-83.

Giné, C. (2004). Servicios y calidad de vida para las personas con discapacidad intelectual. Revista Española sobre Discapacidad Intelectual, 35(2), 1-14.

Gómez, M., Canal., R. \& Verdugo, M. A. (2002). Evaluación de la calidad de vida de adultos con discapacidad intelectual en servicios residenciales comunitarios. Revista de Psicología General y Aplicada, 55(4), 591-602.

Gómez, L., Arias, A., Verdugo, M. \& Navas, P. (2012). An outcomes-based assessment of quality of life in social services. Social Indicators Research, 106(1), 81-93.

Hatton, C., Emerson, E., Robertson, J., Henderson, D. \& Cooper, J. (1995). The quality and costs of residential services for adults with multiple disabilities: A comparative evaluation. Research in Developmental Disabilities, 16(6), 439-460.

Holburn, S., Cea, C. D., Coull, L. \& Goode, D. (2007). What is working and not working: Using focus groups to address quality of life of people living in group homes. Journal of Developmental and Physical Disabilities, 20(1), 1-9.

Keith, R. \& Rawlinson, R. (1998). Quality of life issues in the development and evaluation of services for people with intellectual disability. Journal of Intellectual \& Developmental Disability, 23(3), 199-218.

Kim, S., Larson, A. \& Lakin, C. (2001). Behavioural outcomes of deinstitutionalisation for people with intellectual disability: A review of US studies conducted between 1980 and 1999. Journal of Intellectual \& Developmental Disability, 26(1), 35-50.

Luckasson, R., Borthwick-Duffy, S., Buntinx, W. H. E., Coulter, D. L., Craig, E. M., Reeve, A., et al. (2002). Mental retardation: Definition, classification, and systems of supports (10a. ed.). Washington, DC: American Association on Mental Retardation.
Mayring, P. (2000) Qualitative content analysis. Forum qualitative social research, 1(2). Recuperado el 5 de diciembre de 2010, de http://www.qualitative-research.net/index.php/fqs/article/viewArticle/1089/2385

Mirfin-Veitch, B., Bray, A. \& Ross. N. (2003). "It was the hardest and most painful decision of my life!": Seeking permanent out-of-homeplacement for sons and daughters with intellectual disabilities. Journal of Intellectual $\mathbb{E}$ Developmental Disability, 28(2), 99-111.

O'Brien, P., Thesing, A., Tuck, B. \& Capie, A. (2001). Perceptions of change, advantage and quality of life for people with intellectual disability who left a long stay institution to live in the community. Journal of Intellectual \& Developmental Disability, 26(1), 67-82.

Rouke, A., Grey. I., Fuller, R. \& McClean, B. (2004). Satisfaction with living arrangements of older adults with intellectual disability. Journal of Learning Disabilities, 8(1), 12-29.

Schalock, R. \& Kierman, W. (1990). Habilitation planning for adults with disabilities. New York: Springer Verlag.

Schalock, R., Keith, K., Verdugo, M. \& Gómez, L. (2010). Quality of life model development and use in the field of intellectual disability. En R. Kober (Ed.), Enhancing the quality of life of people with intellectual disability: From theory to practice (pp. 17-32). New York: Springer.

Schalock, R. L. \& Verdugo, M. A. (2003). Calidad de vida. Manual para profesionales de la educación, salud y servicios sociales (M. A. Verdugo \& C. Jenaro, Trads.). Madrid: Alianza Editorial.

Schalock, R. L. \& Verdugo, M. A. (2007). El concepto de calidad de vida en los servicios y apoyos para personas con discapacidad intelectual. Siglo Cero, 38(4), 21-36.

Schalock, R., Verdugo, M. A., Bonham, G., Fontava, S. \& Van Loon, J. (2008). Enhancing personal outcomes: Organizational strategies, guidelines, and examples. Journal of Policy and Practice in Intellectual Disabilities, 5(1), 31-55.

Stancliffe, R. (2002). Provision of residential services for people with intellectual disability in Australia: An 
international comparison. Journal of Intellectual \& Developmental Disability, 27(2), 117-124.

Stancliffe, R. J., Hayden, M. F., Larson, S. \& Lakin, K. C. (2002). Longitudinal study on the adaptive and challenging behaviors of deinstitutionalized adults with intellectual disability. American Journal on Mental Retardation, 107(4), 302-320.

Townsend-White, C., Pham, A. \& Vassos, M. (2012). A systematic review of quality of life measures for people with intellectual disabilities and challenging behaviours. Journal of Intellectual Disability Research, 56(3), 270-284.

Vega, V., Jenaro, C., Cruz, M. \& Flores, N. (2011). Calidad de vida de adultos con discapacidad intelectual en centros residenciales permanentes chilenos. Siglo Cero, 42(2), 26-38.
Vega, V., Jenaro, C., Morillo, M., Cruz, M. \& Flores, N. (2011). Servicios residenciales en Chile, calidad de vida y apoyos: aproximación a una realidad desconocida. Psicología, Conocimiento y Sociedad, 1(3), 52-70.

Verdugo, M. A., Arias, B. \& Ibáñez, A. (2007). SIS. Escala de Intensidad de Apoyos. Madrid: TEA.

Young, L. (2006). Community and cluster centre residential services for adults with intellectual disability: Long-term results from an Australian-matched sample. Journal of Intellectual Disability Research, 50(6), 419-431.

Young, L., Ashman, A., Sigafoos, J. \& Grevell, P. (2001). Closure of Challinor Centre II: An extended report on 95 individuals after 12 months of community living. Journal of Intellectual \& Developmental Disability, 26(1), 51-66. 